\title{
Atributos químicos relacionados ao processo de sulfurização em solos construídos após mineração de carvão
}

\author{
Chemical attributes of constructed soils after surface coal mining related \\ to sulphurization process
}

\author{
Alberto Vasconcellos Inda ${ }^{I^{*}}$ Oscar Rafael Gadea Quinõnes ${ }^{\text {II }}$ Elvio Giasson ${ }^{\text {I }}$ \\ Carlos Alberto Bissani ${ }^{\mathrm{I}}$ Deborah Pinheiro Dick ${ }^{\mathrm{III}}$ Paulo César do Nascimento ${ }^{\mathrm{I}}$
}

\section{RESUMO}

A mineração de carvão a céu aberto envolve a remoção superficial do solo e de camadas geológicas, os quais devem ser repostos visando à reabilitação das áreas mineradas. Essa obra expõe o carvão às condições oxidativas do ar e altera as características originais do solo e da paisagem. Como consequência, o processo de sulfurização pode ser ativado nos solos construídos em razão da oxidação da pirita, condicionando alterações químicas e mineralógicas. Nesse contexto, o estudo objetivou avaliar a evolução temporal de solos construídos há 24 (SA-24) e dois (SA-2) anos, em duas áreas mineradas e reconstruídas no Município de Minas do Leão, Rio Grande do Sul (RS), tendo como base atributos químicos relacionados com o processo de sulfurização. Para tanto, foram determinados o $\mathrm{pH}$, a condutividade elétrica (CE), os teores de bases (SB) e de alumínio $\left(\mathrm{Al}^{3+}\right)$ trocáveis, a acidez potencial $(H+A l)$, os teores totais de Al, Fe e Si e o teor de sulfato solúvel $\left(\mathrm{S}_{-} \mathrm{SO}_{4}{ }^{2-}\right)$ e calculadas a capacidade de troca de cátions (CTC), a saturação por bases (V) e a saturação por alumínio (m). Os resultados indicaram a atuação do processo de sulfurização nos solos construídos de ambas as áreas reabilitadas. A CE e os teores de $\mathrm{S}_{-} \mathrm{SO}_{4}{ }^{2-}$ foram maiores na SA2 , indicando um estádio de sulfurização mais ativo nos solos dessa área. Na SA-24, a menor CE e os menores teores de S$\mathrm{SO}_{4}{ }^{2-}$, de $\mathrm{SB}$ e $\mathrm{V}$, bem como a maior saturação por Al e teores de $H+A l$, sugerem a proximidade do estádio de póssulfurização. Elementos como Al e P concentraram-se relativamente nos solos construídos mais antigos. Os solos em ambas as áreas apresentam limitações químicas para o desenvolvimento de vegetação, influindo negativamente para a recuperação das áreas.

Palavras-chave: sulfurização, acidificação, pirita, áreas degradadas.

\section{ABSTRACT}

Coal stripmining involves the removal of upper soil and geological layers, which must be relocated in a similar way that in the original profile when reconstructing the landscape. As this process changes soil and landscape characteristics, pirite may oxide and change soil chemical and mineralogical characteristics. In this context, the study aimed to evaluate the temporal evolution of soils reconstructed 2 (SA-2) and 24 (SA-24) year ago, in Boa Vista Coal Mining, in Minas do Leão, Rio Grande do Sul, Brazil. Soil characteristics measured were $p H$, electrical conductivity (EC), bases and aluminium content, potential acidity $(H+A l)$, and contents of $\mathrm{Al}, \mathrm{Fe}, \mathrm{Si}$, and soluble sulphate. Calculations accounted for cation exchange capacity, base saturation and aluminum saturation. Results indicated occurrence of oxidation processes in both reconstructed areas. Electrical condutivity and soluble phosphate contents were higher in SA-2, indicating a more active sulphurization stage in these soils. In soils of area SA24, lower EC and smaller contents of soluble sulphate, lower base saturation, as well as higher aluminum saturation and $H+A l$ suggest a more advanced sulphurization process compared to SA-2. Aluminum and P concentrated in older soil profiles. Soils in both areas have chemical limitations for plant growth with adversely affects to recuperation of the area.

Key words: sulphurization, acidification, pyrite, degraded lands.

\section{INTRODUÇÃO}

No processo de mineração de carvão a céu aberto em faixas (stripmining) (KOPPE \& COSTA, 2002),

IDepartamento de Solos, Faculdade de Agronomia, Universidade Federal do Rio Grande do Sul (UFRGS), 90001-970, Porto Alegre, RS, Brasil. E-mail: alberto.inda@ufrgs.br. *Autor para correspondência.

IIPrograma de Pós-graduação em Ciência do Solo, Faculdade de Agronomia, UFRGS, Porto Alegre, RS, Brasil.

IIIDepartamento de Físico-química, Instituto de Química, UFRGS, Porto Alegre, RS, Brasil. 
o carvão é extraído após a remoção e estocagem em separado do solo (horizontes pedogenéticos A, B e C), do saprolito e dos estéreis, compostos pelas camadas geológicas sobrejacentes ou intercaladas à camada de carvão (siltitos, argilitos e folhelhos carbonosos) (PINTO, 1997; KÄMPF et al., 2000). A recuperação de áreas mineradas envolve a reposição dos materiais estéreis e do saprolito na cava minerada e a cobertura destes com o solo retirado no início do processo, visando à reconstituição topográfica (GAIVIZZO et al., 2002). Essas camadas passam a ser denominadas de "solos construídos", pois ocorrem mudanças nas características originais do solo e sobre os quais passam a atuar processos pedogenéticos (KÄMPF et al., 1997; CAMPOS et al., 2003). Quando o processo de reabilitação de áreas mineradas é inadequado, um processo pedogenético previsível nos solos construídos e nas demais áreas afetadas pela mineração de carvão é a sulfurização. Nesses locais, sulfetos (principalmente a pirita $-\mathrm{FeS}_{2}$ ) presentes nos materiais estéreis e resíduos de carvão, quando expostos ao oxigênio e à água, oxidam-se, formando ácido sulfúrico $\left(\mathrm{H}_{2} \mathrm{SO}_{4}\right)($ FANNING \& FANNING, 1989).

Nos solos construídos, a perda de estrutura decorrente de deficiências nos processos de lavra e recuperação das áreas mineradas implica aumento da densidade do solo e diminuição da taxa de infiltração de água, modificações que ocasionam aumento do escoamento superficial e do transporte de solo pela erosão (CIOLKOSZ et al., 1985; POTTER et al., 1988; KÄMPF et al., 2000; SHUKLA et al., 2004; QUIÑONES et al., 2008). Como consequência da erosão em solos construídos, materiais de camadas subsuperficiais contendo pirita são expostos a condições ambientais favoráveis a sua oxidação, ativando assim o processo de sulfurização. A intensa acidificação produzida, caracterizada por valores de $\mathrm{pH}$ inferiores a 3,5, determina a dissolução de minerais aluminossilicatados, bem como a formação de novas fases minerais a partir dos produtos dessa dissolução (KÄMPF et al., 1997). A evolução desse processo em solos construídos também é representada por decréscimos dos teores de cátions básicos e aumentos de alumínio trocável, sulfato solúvel e da condutividade elétrica, comparativamente às condições do solo natural anteriormente à mineração (KÄMPF et al., 1997; CAMPOS et al., 2003), e tais alterações são dependentes e indicativas do estádio de sulfurização alcançado pelo solo (KÄMPF et al., 1997).

A legislação ambiental requer $o$ estabelecimento de um plano de execução de recuperação ambiental das áreas mineradas, em que os solos construídos devem proporcionar condições para o desenvolvimento de vegetação (PINTO, 1997). O presente estudo objetivou avaliar a evolução temporal de solos construídos há 24 e dois anos no Município de Minas do Leão, Rio Grande do Sul (RS), tendo como base atributos químicos desses solos relacionados ao processo pedogenético de sulfurização.

\section{MATERIAL E MÉTODOS}

O trabalho foi realizado na mina de carvão Boa Vista, da Companhia Riograndense de Mineração (CRM), situada em região próxima à margem do Km 85 da BR-290, no Município de Minas do Leão (RS) (Figura 1). As camadas de carvão fazem parte da formação Rio Bonito, do grupo Guatá, do supergrupo Tubarão e do Permiano Superior (BRASIL, 1986). No processo de construção dos solos na mina Boa Vista, os materiais geológicos foram repostos em camadas, por tratores de esteira, para preenchimento das cavas mineradas. Como cobertura final, foi usado material do solo natural (mistura de horizontes A, B e C), que fora removido e separado no início do processo de mineração. Após a realização da recomposição topográfica, foi implantada a cobertura vegetal, com utilização de várias espécies de gramíneas como a Bracchiaria humidícola, Bracchiaria brizanta, Bracchiaria dyctomeura, Panicum maximum, entre outras. Uma vez estabelecida a vegetação de cobertura, foram implantadas as espécies vegetais de reflorestamento Eucaliptus ssp. e Pinus ssp.

Foram selecionados perfis em duas áreas da mina reabilitadas em 1978 e 2000 . Essas áreas representavam, na época de amostragem, solos construídos com idades de 24 (SA-24) e dois (SA-2) anos de construção. Na área SA-24, revegetada com Eucaliptus ssp., Pinus ssp. e gramíneas, foram selecionados quatro perfis de solos construídos, identificados como: P1-24, em uma trincheira aberta num reflorestamento de eucaliptos; P2-24, em uma trincheira aberta em área de capoeira com evidência de alta erosão; P3-24 e P4-24, em trincheiras abertas em reflorestamento de pinus. Na área SA-2, revegetada com gramíneas e com Eucaliptus ssp., ambas com desenvolvimento heterogêneo e incipiente, foram selecionados três perfis de solos construídos ao longo de uma sequência em declive, identificados como: P1-2, na porção inferior; P2-2, na porção média; e P3-2, na porção superior. A separação das camadas ou horizontes nos perfis selecionados e a coleta de amostras em todas as camadas ou horizontes foram realizadas conforme LEMOS \& SANTOS (1996). A caracterização morfológica e física desses perfis de solos construídos, além de atributos químicos relacionados à composição dos materiais utilizados na construção desses solos 


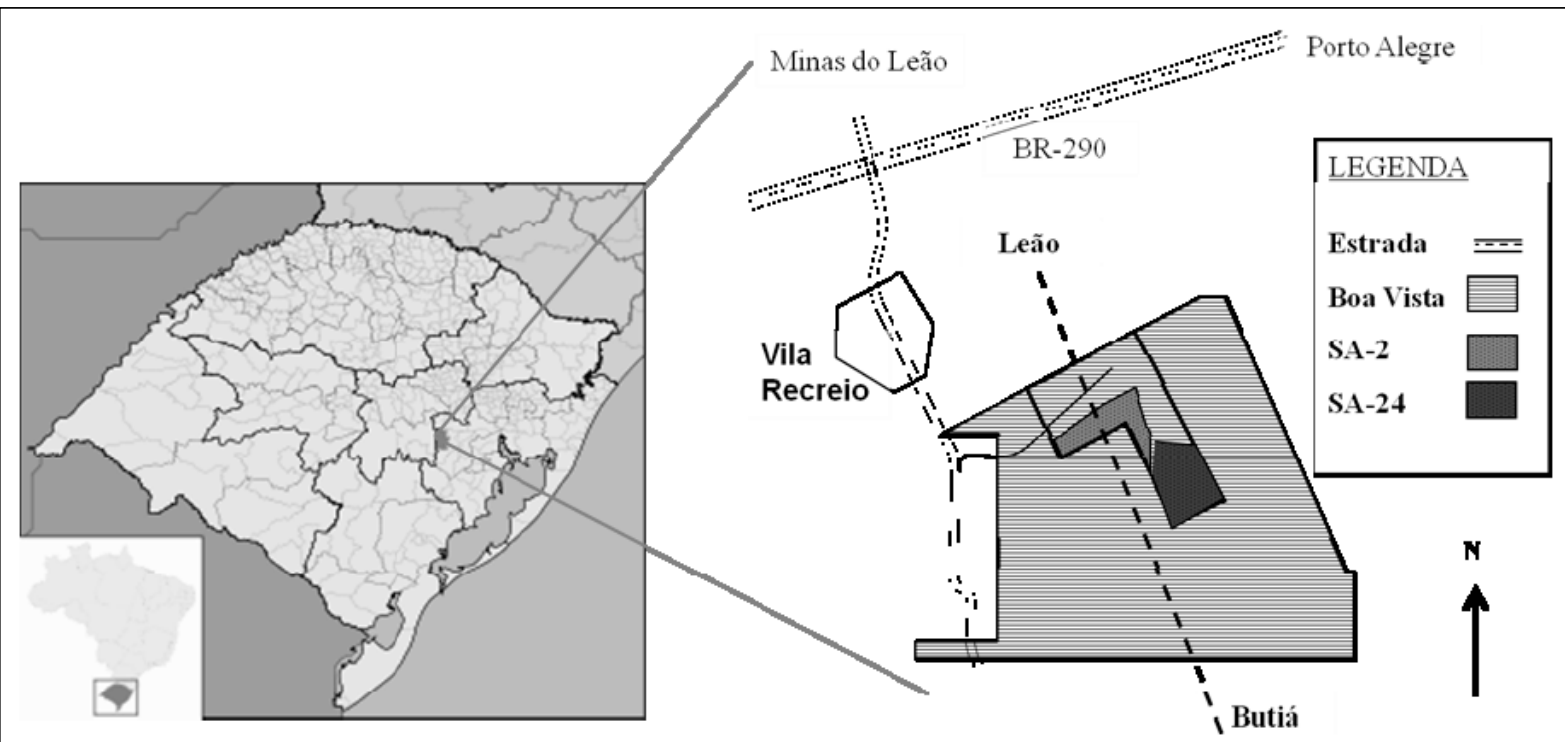

Figura 1 - Localização e vias de acesso à mina de carvão Boa Vista. (SA-2: solos construídos há dois anos; SA-24: solos construídos há 24 anos).

(CTC, C orgânico, etc...), é descrita por QUIÑONES et al. (2008).

A determinação do $\mathrm{pH}$ e da condutividade elétrica foi realizada no mesmo dia da coleta das amostras. As demais análises foram realizadas em amostras secas ao ar, moídas e passadas em peneiras de $2 \mathrm{~mm}$. As análises químicas consistiram em: $\mathrm{pH}$ em $\mathrm{H}_{2} \mathrm{O}$, na proporção em peso 1:1 de solo em solução (EMBRAPA, 1997), condutividade elétrica (CE), na proporção 1:5 de solo em solução (TEDESCO et al., 1995); teores de bases trocáveis e acidez potencial (EMBRAPA, 1997); alumínio trocável utilizando metodologia indicada para solos com alto teor de carbono orgânico (McLEAN, 1965); teores de Si, Fe, Al e P mediante ataque sulfúrico (EMBRAPA, 1997); teor de sulfato solúvel em $\mathrm{CaCl}_{2} 0,1 \mathrm{~mol} \mathrm{~L}^{-1}$ (BISSANI et al., 1995). Foram calculados os valores de soma de bases, saturação por bases e saturação por alumínio. Para análise dos resultados, foram utilizados parâmetros de estatística descritiva, empregando o programa Microsoft Excel. Para avaliar se existem diferenças significativas entre os atributos analisados nas amostras dos perfis representativos das áreas SA-2 e SA-24, foi aplicado o teste não paramétrico ou de distribuição livre U de Mann-Whitney, segundo KÄMPF et al. (1997), sendo utilizado o programa STATISTIX7 (2000). Esse teste não utiliza valores médios em seu cálculo, mas verifica se as distribuições de probabilidade de uma variável podem ser consideradas iguais nas duas populações de amostras em estudo (SIEGEL, 1975).

\section{RESULTADOS E DISCUSSÃO}

Os atributos químicos avaliados nos perfis variaram irregularmente com a profundidade em ambas as áreas, indicando deficiências na seleção prévia e na reposição dos materiais nas cavas de mineração (Tabelas 1 e 2). O pH não diferiu significativamente entre os conjuntos de camadas das áreas SA-24 e os SA-2 (Tabela 3), apresentando valores médios de 4,6 e 4,8 , respectivamente. Essa semelhança evidencia um processo já intenso de oxidação da pirita na área reabilitada há dois anos (SA-2), embora algumas de suas camadas apresentem valores de $\mathrm{pH}$ mais alcalino (C1 e C2 do P2-2; C2 e C3 do P3-2) (Tabela 1), os quais representam ainda características do material geológico empregado na construção. A rápida acidificação constatada na SA-2 é consequência da oxidação da pirita exposta à superfície por um processo erosivo acelerado, favorecido pela declividade acentuada e, praticamente, ausência de cobertura vegetal do solo (QUIÑNONES et al., 2008).

A condutividade elétrica diferiu significativamente entre as áreas SA-24 e SA-2, com valores médios de $101,0 \mu \mathrm{S} \mathrm{cm} \mathrm{cm}^{-1}$ e $240,6 \mu \mathrm{S} \mathrm{cm}^{-1}$, respectivamente. Segundo DANIELS (1996), valores $\mathrm{CE}>100 \mu \mathrm{S} \mathrm{cm}^{-1}$ indicam restrição ao desenvolvimento de espécies sensíveis ao excesso de sais. Da mesma forma, os teores de sulfato solúvel $\left(\mathrm{S}_{-} \mathrm{SO}_{4}{ }^{2-}\right)$ diferiram significativamente entre as áreas, com valores médios de $0,17 \mathrm{~g} \mathrm{~kg}^{-1}$ para SA-24 e $0,38 \mathrm{~g} \mathrm{~kg}^{-1}$, para a SA-2 (Tabela 1). A expressiva correlação obtida neste estudo entre 
Atributos químicos relacionados ao processo de sulfurização em solos construídos após mineração de carvão.

1063

Tabela 1 - Atributos químicos dos perfis de solos construídos com dois e 24 anos de construção.

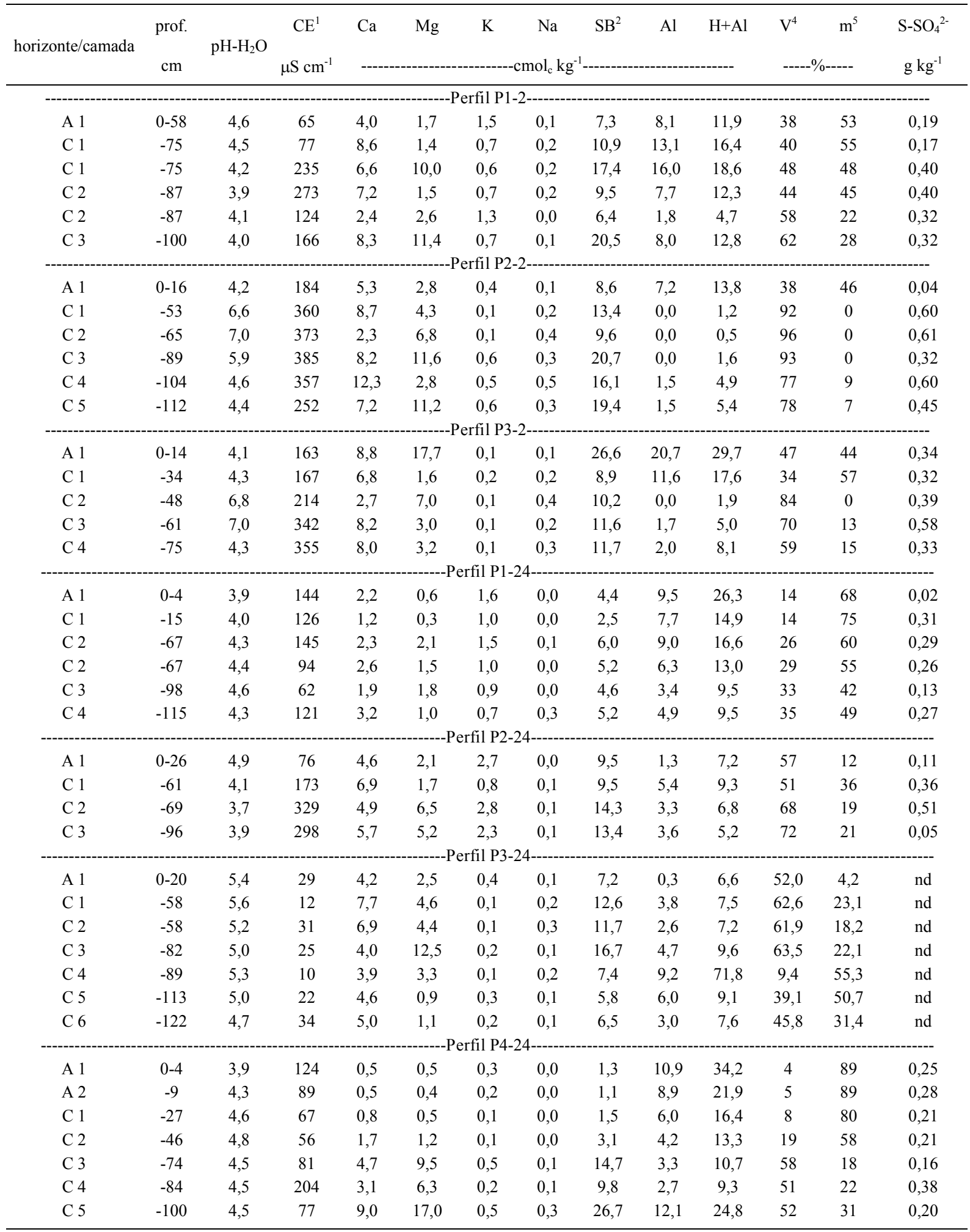

${ }^{1}$ Condutividade elétrica; ${ }^{2}$ Soma de bases; ${ }^{3}$ Capacidade de troca de cátions; ${ }^{4}$ Saturação por bases;

${ }^{5}$ Saturação por alumínio; ${ }^{6}$ Não determinado. 
Tabela 2 - Teores totais de elementos extraídos por ataque sulfúrico e índice de intemperismo $\mathrm{Ki}$ das camadas dos perfis de solos construídos com dois e 24 anos de construção.

\begin{tabular}{|c|c|c|c|c|c|c|}
\hline $\begin{array}{l}\text { Horizonte } \\
\text { /camada }\end{array}$ & $\begin{array}{l}\text { prof. } \\
\mathrm{cm}\end{array}$ & $\mathrm{SiO}_{2}$ & \multicolumn{4}{|c|}{ 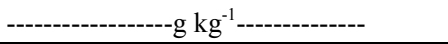 } \\
\hline \multicolumn{7}{|c|}{-----------------------------Perfil P1-2----------------------------- } \\
\hline A 1 & $0-58$ & 164,8 & 43,5 & 93,2 & 0,2 & 3,0 \\
\hline $\mathrm{C} 1$ & -75 & 272,9 & 52,5 & 137,4 & 0,1 & 3,4 \\
\hline $\mathrm{C} 1$ & -75 & 286,3 & 34,5 & 150,6 & 0,0 & 3,2 \\
\hline C 2 & -87 & 277,3 & 31,7 & 157,2 & 0,3 & 3,0 \\
\hline C 2 & -87 & 112,4 & 47,3 & 71,8 & 0,2 & 2,6 \\
\hline C 3 & -100 & 263,9 & 45,6 & 150,6 & 0,3 & 3,0 \\
\hline \multicolumn{7}{|c|}{ 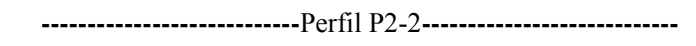 } \\
\hline A 1 & $0-16$ & 191,5 & 45,3 & 99,3 & 0,5 & 3,3 \\
\hline C 1 & -53 & 302,0 & 28,7 & 168,8 & 0,3 & 3,0 \\
\hline $\mathrm{C} 2$ & -65 & 306,4 & 66,8 & 142,3 & 0,5 & 3,7 \\
\hline C 3 & -89 & 304,1 & 18,6 & 180,3 & 0,4 & 2,9 \\
\hline $\mathrm{C} 4$ & -104 & 295,3 & 63,6 & 132,4 & 0,7 & 3,8 \\
\hline C 5 & -112 & 235,4 & 46,6 & 102,6 & 0,6 & 3,9 \\
\hline \multicolumn{7}{|c|}{-----Perfil P3-2---------- } \\
\hline A 1 & $0-14$ & 351,2 & 82,8 & 160,6 & 0,7 & 3,7 \\
\hline C 1 & -34 & 323,1 & 63,1 & 154,0 & 0,7 & 3,6 \\
\hline $\mathrm{C} 2$ & -48 & 346,7 & 50,6 & 107,5 & 0,6 & 5,5 \\
\hline C 3 & -61 & 337,7 & 15,7 & 119,2 & 0,4 & 4,8 \\
\hline $\mathrm{C} 4$ & -75 & 308,6 & 25,2 & 109,2 & 0,6 & 4,8 \\
\hline \multicolumn{7}{|c|}{----Perfil P1-24---------- } \\
\hline A 1 & $0-4$ & 214,6 & 39,3 & 140,6 & 1,3 & 2,6 \\
\hline C 1 & -15 & 217,0 & 87,1 & 137,4 & 1,1 & 2,7 \\
\hline C 2 & -67 & 263,9 & 97,7 & 167,1 & 0,9 & 2,7 \\
\hline C 2 & -67 & 223,6 & 98,8 & 147,2 & 1,0 & 2,6 \\
\hline C 3 & -98 & 186,6 & 83,9 & 130,8 & 1,2 & 2,4 \\
\hline $\mathrm{C} 4$ & -115 & 322,1 & 49,6 & 171,5 & 0,5 & 3,2 \\
\hline \multicolumn{7}{|c|}{----Perfil P2-24------ } \\
\hline A 1 & $0-26$ & 163,5 & 42,8 & 88,7 & 0,9 & 3,1 \\
\hline C 1 & -61 & 295,3 & 48,6 & 154,0 & 0,4 & 3,3 \\
\hline C 2 & -69 & 288,7 & 18,9 & 177,1 & 0,5 & 2,8 \\
\hline C 3 & -96 & 277,3 & 28,5 & 183,7 & 0,3 & 2,6 \\
\hline \multicolumn{7}{|c|}{-----Perfil P3-24----------- } \\
\hline A 1 & $0-20$ & 211,2 & 30,3 & 72,9 & 1,1 & 4,9 \\
\hline C 1 & -58 & 348,8 & 47,0 & 114,1 & 0,6 & 5,2 \\
\hline C 2 & -58 & 335,6 & 21,0 & 86,1 & 0,4 & 6,6 \\
\hline C 3 & -82 & 259,4 & 97,7 & 165,4 & 1,2 & 2,7 \\
\hline C 4 & -89 & 186,6 & 28,6 & 107,5 & 1,2 & 2,9 \\
\hline C 5 & -113 & 357,8 & 23,3 & 168,8 & 0,6 & 3,6 \\
\hline C 6 & -122 & 357,8 & 16,0 & 197,0 & 0,7 & 3,1 \\
\hline \multicolumn{7}{|c|}{----Perfil P4-24--------- } \\
\hline A 1 & $0-4$ & 406,1 & 36,3 & 194,6 & 1,1 & 3,5 \\
\hline A 2 & -9 & 317,8 & 56,6 & 190,8 & 1,6 & 2,8 \\
\hline C 1 & -27 & 279,5 & 66,9 & 171,7 & 0,9 & 2,8 \\
\hline $\mathrm{C} 2$ & -46 & 300,1 & 68,0 & 168,5 & 0,9 & 3,0 \\
\hline C 3 & -74 & 341,3 & 47,9 & 181,2 & 0,4 & 3,2 \\
\hline C 4 & -84 & 306,0 & 57,1 & 162,4 & 0,3 & 3,2 \\
\hline C 5 & -100 & 409,0 & 60,7 & 157,9 & 0,3 & 4,4 \\
\hline
\end{tabular}

$\mathrm{Ki}=\mathrm{SiO}_{2} / \mathrm{Al}_{2} \mathrm{O}_{3}$.

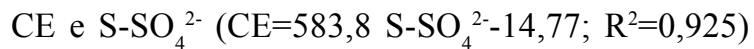
confirma que a $\mathrm{CE}$ pode ser utilizada como indicativo da formação de sulfatos solúveis durante o processo de sulfurização, conforme já constatado por KÄMPF et al. (1997) em solos construídos no Rio Grande do Sul. A maior CE nos solos da área SA-2 está relacionada ao estádio de sulfurização ativa, em que a liberação de sais para a solução do solo é intensa (FANNING \& FANNING, 1989; ALMEIDA, 1999; KÄMPF etal., 2000). Solos construídos mais antigos, em estádio de póssulfurização, tendem a uma diminuição dos valores de $\mathrm{CE}$ em razão do gradativo esgotamento das fontes de sais, da lixiviação destes (SCHAFER et al., 1980; CIOLKOSZ et al., 1985) e da precipitação de novas fases minerais pela neogênese (KÄMPF et al., 1997).

Em relação aos atributos químicos relacionados ao complexo de troca, os teores de $\mathrm{Ca}$, $\mathrm{Mg}, \mathrm{Na}$, a acidez potencial $(\mathrm{H}+\mathrm{Al})$ e os valores de soma de bases (SB), saturação por bases (V) e saturação por alumínio $(\mathrm{m})$ diferiram significativamente entre as áreas SA-2 e SA-24; no entanto, esse resultado não foi constatado para os teores de $\mathrm{Ke} \mathrm{Al}$ (Tabela 3). O valor médio da soma de bases (SB), com predomínio de Ca e $\mathrm{Mg}$, foi superior na área SA-2 $\left(13,4 \mathrm{cmol}_{\mathrm{c}} \mathrm{kg}^{-1}\right)$, o que conferiu também um maior valor médio de $\mathrm{V}(63 \%)$ comparativamente à área SA-24 $\left(\mathrm{SB}=8,4 \mathrm{cmol}_{\mathrm{c}} \mathrm{kg}^{-1}\right.$; $\mathrm{V}=39 \%$ ) (Tabela 1). Esses resultados estão de acordo os obtidos por HAERING et al. (1993) em solos construídos jovens, no Estado de Virginia, Estados Unidos, e confirmam as constatações feitas por QUIÑONES et al. (2008), que indicaram para a área SA2 um processo mais ativo de dissolução protônica de minerais tamponantes (carbonatos e esmectitas) constituintes das camadas geológicas utilizadas na recuperação dessas áreas (PINTO, 1997; KÄMPF et al., 2000).

A deficiência de cátions básicos em solos construídos após mineração de carvão reflete a intensa intemperização dos minerais e as consequentes perdas desses elementos por lixiviação (PITCHEL et al., 1994). Portanto, os menores valores de SB e V na área SA-24 devem-se ao maior tempo de exposição das camadas contendo pirita a condições oxidantes (sulfurização). Essa situação favorece a dissolução de silicatos e a lixiviação de cátions (Ca, $\mathrm{Mg}$ e $\mathrm{Na}$ ) acompanhados do ânion sulfato (CAMPOS et al., 2003), bem como a já referida neogênese de fases minerais contendo $\mathrm{Ke} \mathrm{Na}$ (KÄMPF et al., 1997).

A maior acidez potencial $(\mathrm{H}+\mathrm{Al})$ na SA-24 $\left(15,4 \mathrm{cmol}_{\mathrm{c}} \mathrm{kg}^{-1}\right)$, em comparação com a SA-2 $\left(9,4 \mathrm{cmol}_{\mathrm{c}}\right.$ $\mathrm{kg}^{-1} ; \mathrm{P}=0,0672$ ), é decorrente da dissolução dos silicatos, que resulta em elevada liberação de prótons $\left(\mathrm{H}^{+}\right)$na hidrólise do $\mathrm{Al}$ e $\mathrm{Fe}$ liberados no processo 
Atributos químicos relacionados ao processo de sulfurização em solos construídos após mineração de carvão.

Tabela 3 - Parâmetros estatísticos relacionados à evolução dos conjuntos de amostras dos solos construídos com dois e 24 anos de construção.

\begin{tabular}{|c|c|c|c|c|c|c|}
\hline Atributos do solo & $\begin{array}{l}\text { Idade } \\
\text { anos }\end{array}$ & Média & Mínimo & Máximo & $\mathrm{U}^{3}$ & $\mathrm{p}$ \\
\hline \multirow{2}{*}{$\mathrm{pH}$} & 2 & 4,8 & 3,9 & 7,0 & 217,5 & 0,9797 \\
\hline & 24 & 4,6 & 3,7 & 5,6 & 214,5 & \\
\hline \multirow{2}{*}{$\mathrm{CE}, \mu \mathrm{S} \mathrm{cm}{ }^{-1}$} & 2 & 241 & 65 & 385 & 372,0 & 0,0001 \\
\hline & 24 & 101 & 10 & 329 & 60,0 & \\
\hline \multirow{2}{*}{$\mathrm{Ca}, \mathrm{cmol}_{\mathrm{c}} \mathrm{kg}^{-1}$} & 2 & 7,0 & 2,3 & 12,3 & 349,0 & 0,0008 \\
\hline & 24 & 3,8 & 0,5 & 9,0 & 83,0 & \\
\hline \multirow{2}{*}{$\mathrm{Mg}, \mathrm{cmol}_{\mathrm{c}} \mathrm{kg}^{-1}$} & 2 & 5,7 & 1,4 & 17,7 & 295,5 & 0,0446 \\
\hline & 24 & 3,7 & 0,3 & 17,0 & 136,5 & \\
\hline \multirow{2}{*}{$\mathrm{K}, \mathrm{cmol}_{\mathrm{c}} \mathrm{kg}^{-1}$} & 2 & 0,5 & 0,1 & 1,5 & 186,0 & 0,4485 \\
\hline & 24 & 0,8 & 0,1 & 2,8 & 246,0 & \\
\hline \multirow{2}{*}{$\mathrm{Na}, \mathrm{cmol}_{\mathrm{c}} \mathrm{kg}^{-1}$} & 2 & 0,2 & 0,0 & 0,5 & 339,5 & 0,0013 \\
\hline & 24 & 0,1 & 0,0 & 0,3 & 92,5 & \\
\hline \multirow{2}{*}{$\mathrm{SB}, \mathrm{cmol}_{\mathrm{c}} \mathrm{kg}^{-1}$} & 2 & 13,4 & 6,4 & 26,6 & 326,5 & 0,0052 \\
\hline & 24 & 8,4 & 1,1 & 26,7 & 105,5 & \\
\hline \multirow{2}{*}{$\mathrm{Al}, \mathrm{cmol}_{\mathrm{c}} \mathrm{kg}^{-1}$} & 2 & 5,6 & 0,0 & 20,7 & 175,5 & 0,3090 \\
\hline & 24 & 5,5 & 0,3 & 12,1 & 256,5 & \\
\hline \multirow{2}{*}{$\mathrm{H}+\mathrm{Al}, \mathrm{cmol}_{\mathrm{c}} \mathrm{kg}^{-1}$} & 2 & 9,4 & 0,5 & 29,7 & 143,5 & 0,0672 \\
\hline & 24 & 15,4 & 5,2 & 71,8 & 288,5 & \\
\hline \multirow{2}{*}{$\mathrm{V}, \%$} & 2 & 63 & 34 & 96 & 330,0 & 0,0039 \\
\hline & 24 & 39 & 4 & 72 & 102,0 & \\
\hline \multirow{2}{*}{$\mathrm{m}, \%$} & 2 & 25 & 0 & 57 & 122,0 & 0,0174 \\
\hline & 24 & 43 & 4 & 89 & 310,0 & \\
\hline \multirow{2}{*}{$\mathrm{S}-\mathrm{SO}_{4}^{2-}, \mathrm{g} \mathrm{kg}^{-1}$} & 2 & 0,38 & 0,04 & 0,61 & 279,0 & 0,0011 \\
\hline & 24 & 0,17 & 0,00 & 0,51 & 63,0 & \\
\hline \multirow{2}{*}{$\mathrm{Fe}_{2} \mathrm{O}_{3}, \mathrm{~g} \mathrm{~kg}^{-1}$} & 2 & 44,9 & 15,7 & 82,8 & 185,0 & 0,4382 \\
\hline & 24 & 52,1 & 16,0 & 98,8 & 247,0 & \\
\hline \multirow{2}{*}{$\mathrm{Al}_{2} \mathrm{O}_{3}, \mathrm{~g} \mathrm{~kg}^{-1}$} & 2 & 130,4 & 71,8 & 180,3 & 129,0 & 0,0279 \\
\hline & 24 & 151,5 & 72,9 & 197,0 & 303,0 & \\
\hline \multirow{2}{*}{$\mathrm{SiO}_{2}, \mathrm{~g} \mathrm{~kg}^{-1}$} & 2 & 274,0 & 112,4 & 351,2 & 200,5 & 0,7030 \\
\hline & 24 & 286,2 & 163,5 & 409,0 & 231,5 & \\
\hline \multirow{2}{*}{$\mathrm{Ki}$} & 2 & 3,62 & 2,60 & 5,50 & 294,5 & 0,0469 \\
\hline & 24 & 3,33 & 2,40 & 6,60 & 137,5 & \\
\hline \multirow{2}{*}{$\mathrm{P}_{2} \mathrm{O}_{5}, \mathrm{~g} \mathrm{~kg}^{-1}$} & 2 & 0,44 & 0,01 & 0,80 & 97,0 & 0,0025 \\
\hline & 24 & 0,81 & 0,30 & 1,60 & 335,0 & \\
\hline
\end{tabular}

${ }^{1}$ Desvio padrão; ${ }^{2}$ Coeficiente de variação; ${ }^{3}$ Teste Mann-Whitney; CE - Condutividade elétrica; SB - Soma de bases; CTC - Capacidade de troca de cátions; V - Saturação por bases; S-Al - Saturação por alumínio. 
(KÄMPF et al., 2000). Os teores médios de Al trocável verificados em ambas as áreas $\left(\mathrm{SA}-245,5 \mathrm{cmol}_{\mathrm{c}} \mathrm{kg}^{-1} \mathrm{e}\right.$ $\mathrm{SA}-2=5,6 \mathrm{cmol}_{\mathrm{c}} \mathrm{kg}^{-1}$ ) representam níveis tóxicos para o desenvolvimento de várias espécies vegetais (Tabela 1). Embora o Al trocável não tenha discriminado as áreas quanto ao estádio do processo de sulfurização, a saturação por $\mathrm{Al}$ diferiu significativamente $(\mathrm{P}=0,0174)$, com valores médios de $43 \%$ para a SA-24 e $25 \%$ para a SA-2. Isso provavelmente se deve à baixa mobilidade do $\mathrm{Al}$, que ocupa os sítios de troca em substituição aos cátions básicos que foram lixiviados nos solos construídos da SA-24. Na SA-2, esse efeito pode ser observado nas camadas superficiais, em razão da maior exposição destas à ação da água e do oxigênio, favorecendo o processo de sulfurização. Já nas camadas subsuperficiais dos mesmos perfis (principalmente P2-2 e P3-2), esse processo foi menos intenso em razão do aumento da compactação (SOARES et al., 2002; QUIÑONES et al., 2008) e da diminuição da condutividade hidráulica (KÄMPF et al., 1997; QUIÑONES et al., 2008). Esses processos restringem a ação da água e do oxigênio, promovendo o aumento da taxa das reações produtoras de alcalinidade, ao mesmo tempo em que retardada a cinética das reações produtoras de acidez (SOARES at al., 2002).

No extrato sulfúrico, os teores médios de $\mathrm{Al}_{2} \mathrm{O}_{3}\left(\mathrm{SA}-24=151,6 \mathrm{~g} \mathrm{~kg}^{-1}\right.$ e SA-2 $\left.=130,4 \mathrm{~g} \mathrm{~kg}^{-1}\right)$ diferiram significativamente $(\mathrm{P}=0,0279)$ entre as áreas, ao contrário do verificado para os teores médios de $\mathrm{Fe}_{2} \mathrm{O}_{3}$ $\left(\mathrm{SA}-24=52,2 \mathrm{~g} \mathrm{~kg}^{-1}\right.$ e SA-2 $\left.=45,0 \mathrm{~g} \mathrm{~kg}^{-1}\right)$ e $\mathrm{SiO}_{2}\left(\mathrm{SA}^{3}\right.$ $24=286,2 \mathrm{~g} \mathrm{~kg}^{-1}$ e SA-2 $=274,0 \mathrm{~g} \mathrm{~kg}^{-1}$ ) (Tabela 2). Os teores significativos mais elevados de $\mathrm{Al}_{2} \mathrm{O}_{3}$ na SA-24 indicam que, uma vez liberado pela dissolução ácida dos silicatos, a baixa mobilidade do $\mathrm{Al}^{3+}$ favorece a sua concentração relativa em razão lixiviação preferencial dos cátions básicos. Os valores da relação molar entre $\mathrm{SiO}_{2} \mathrm{e}_{2} \mathrm{O}_{3}$ (índice $\mathrm{Ki}$ ), utilizada como discriminante de classes de solos em sistemas de classificação taxonômica de solos (EMBRAPA, 2006), apresentaram uma ampla variação nas áreas estudadas $(2,4<\mathrm{Ki}<6,6)$, indicando camadas formadas por materiais de distintos graus de intemperismo. Os valores médios de Ki (SA24=3,3 e SA-2=3,6) indicam uma presença significativa de argilominerais 2:1 em ambas as áreas e, para o conjunto das camadas de cada área, um estágio de intemperização mais avançado na SA-24.

Os teores de $\mathrm{P}_{2} \mathrm{O}_{5}$ apresentaram uma variação de $<0,01$ a $1,6 \mathrm{~g} \mathrm{~kg}^{-1}$, com valores médios de $0,81 \mathrm{~g} \mathrm{~kg}^{-1}$ na SA-24 e $0,44 \mathrm{~g} \mathrm{~kg}^{-1}$ na SA-2. Embora esses teores de $\mathrm{P}_{2} \mathrm{O}_{5}$ sejam considerados baixos, verificouse uma diferença significativa $(\mathrm{P}=0,0025)$ destes entre as áreas, e os maiores teores obtidos na SA-24 também estão relacionados a uma concentração relativa de $\mathrm{P}_{2} \mathrm{O}_{5}$ nessa área, conforme verificado para os teores $\operatorname{de~}_{2} \mathrm{Ol}_{3}$.
Os atributos químicos dos solos construídos avaliados neste estudo indicam que o processo de sulfurização constitui-se como o principal elemento da evolução pedogenética desses solos. Os resultados são suficientes para afirmar que os solos construídos na SA-2 se apresentam em estádio de sulfurização ativa e menos evoluídos em comparação aos solos da SA-24 que se mostram próximos ao estádio de pós-sulfurização. Essa avaliação indica sérias deficiências no processo de construção dos solos realizado em ambas as áreas, cujas consequências ambientais desfavoráveis foram a restrição ao desenvolvimento vegetal adequado, o processo de erosão acelerado e a contaminação do solo e da água. A prévia seleção dos materiais a serem utilizados na construção dos solos, bem como a disposição uniforme destes durante o processo de construção, são medidas que devem ser consideradas na reabilitação de áreas após a mineração de carvão a céu aberto.

\section{CONCLUSÃO}

Os atributos químicos dos perfis de solos em áreas reconstituídas após mineração de carvão foram influenciadas pelo processo de sulfurização e pelo tempo de atuação desse processo. $\mathrm{Na}$ área mais jovem (SA-2), o estádio de sulfurização ativo está representado por valores de $\mathrm{pH}$ muito baixos, associados a uma maior condutividade elétrica, teores elevados de sulfato solúvel e de soma de bases e maior saturação por bases. Na área mais antiga (SA-24), a proximidade do estádio de pós-sulfurização é indicada pela menor condutividade elétrica e pelos menores teores de sulfato solúvel, associados às maiores saturação por alumínio e acidez potencial e teores elevados de alumínio e de fósforo total. Em ambas as áreas, os solos apresentam limitações químicas para o desenvolvimento de vegetação, influindo negativamente para a recuperação dessas áreas e de áreas adjacentes.

\section{REFERÊNCIAS}

ALMEIDA, P.S.G. Acidificação experimental em materiais estéreis de área de mineração de carvão. 1999. $118 \mathrm{f}$. Dissertação (Mestrado em Agronomia - Ciência do solo) Universidade Federal do Rio Grande do Sul, Porto Alegre, RS.

BISSANI, C.A. et al. Determinação de sulfato solúvel em solos tiomórficos de áreas de mineração de carvão. In: CONGRESSO BRASILEIRO DE CIÊNCIA DO SOLO, 25., 1995, Viçosa, MG. Anais... Viçosa: Sociedade Brasileira de Ciência do Solo, 1995. p. 1535-1537.

Ciência Rural, v.40, n.5, mai, 2010. 
BRASIL. Instituto Brasileiro de Geografia e Estatística. Levantamento de recursos naturais. Rio de Janeiro, 1986. V.33, 796p

CAMPOS, M.L. et al. Avaliação de três áreas de solo construído após mineração de carvão a céu aberto em Lauro Müller, Santa Catarina. Revista Brasileira de Ciência do Solo, Viçosa, v.27, p.1123-1137, 2003. Disponível em: <http:/www.scielo.br/ scielo.php? script=s ci_art text \& pid = S $0100-$ $06832003000600017 \& \operatorname{lng}=\mathrm{en} \& \mathrm{nrm}=\mathrm{iso} \& \mathrm{t} \operatorname{lng}=\mathrm{pt}>$. Acesso em: 30 mar. 2010. doi: 10.1590/S0100-06832003000600017.

CIOLKOSZ, E.J. et al. Characteristics, genesis, and classification of Pennsylvania minesoils. Soil Science, Philadelphia, v.139, n.3, p.232-238, 1985. Disponível em: <http://soil.scijournals.org/ cgi/reprint/72/1/231>. Acesso em: 30 mar. 2010. doi:10.2136/ sssaj2007.0047

DANIELS, W.L. Manipulating the chemical properties of soil and mining wastes. In: ALVAREZ V.V.H. et al. (Eds.). O solo nos grandes domínios morfoclimáticos do Brasil e o desenvolvimento sustentado. Viçosa: Sociedade Brasileira de Ciência do Solo, 1996. p.869-897.

EMBRAPA. Serviço Nacional de Levantamento e Conservação de Solos. Manual de métodos de análise de solo. 2.ed. Rio de Janeiro, 1997. 212p.

EMBRAPA. Sistema Brasileiro de Classificação de Solos 2.ed. Brasília: Embrapa Solos, 2006. 306p.

FANNING, D.S.; FANNING, M.C.B. Soil: morphology, genesis and classification. New York: J. Willey, 1989. 395p.

GAIVIZZO L.H.B. et al. Potencial poluidor de regiões carboníferas. II-Efeitos da recuperação com camadas de solo sobre as plantas e a população microbiana. Ciência Rural, Santa Maria, v.32, p.955-961, 2002. Disponível em: $<$ http:// www.scielo.br/scielo.php? script $=$ sci arttext\&pid $=\mathrm{S} 0103$ $84782002000600007 \& \operatorname{lng}=$ en $\& n r m=i s o>$. Acesso em: 30 mar. 2010. doi: $10.1590 / \mathrm{S} 0103-84782002000600007$

HAERING, K.C. et al. Changes in mine soil properties resulting from overburden weathering. Journal of Environmental Quality, Madison, v.22, p.194-200, 1993.

KÄMPF, N. et al. Propriedades, pedogênese e classificação de solos construídos em áreas de mineração na bacia carbonífera do baixo Jacuí (RS). Revista Brasileira de Ciência do Solo, Viçosa, v.21, p.79-88, 1997.

KÄMPF, N. et al. Solos construídos em áreas de mineração da Bacia Carbonífera. In: Centro de Ecologia - UFRGS. Carvão e meio ambiente. Porto Alegre: UFRGS, 2000. Cap.17, p. 596-640.
KOPPE, J.C.; COSTA, J.F.C. Mineração. In: TEIXEIRA, E.C. Meio ambiente e carvão: impactos da exploração e utilização. Porto Alegre: FEPAM, 2002. p.15-27.

LEMOS, R.C.; SANTOS, R.D. Manual de descrição e coleta de solos no campo. 3.ed. Campinas: Sociedade Brasileira de Ciência do Solo, 1996. 84p.

McLEAN, E.O. Aluminum. In: BLACK, C.A. et al. Methods of soil analysis. Part. 2. Madison: ASA, 1965. p. 978-998. (Agronomy Series 9).

PINTO, L.F.S. Potencial de acidificação e neutralização dos materiais geológicos para a composição do solo construído em áreas de mineração de carvão. 1997. $186 f$. Tese (Doutorado em Agronomia - Ciências do Solo) Universidade Federal do Rio Grande do Sul, Porto Alegre, RS

PITCHEL, J.R. et al. Comparison amendments and management practices for long-term reclamation of abandoned mine lands. Journal of Environmental Quality, Madison, v.23, p.766-772, 1994.

POTTER, K.N. et al. Physical properties of constructed and undisturbed soils. Soil Science Society America Journal, Madison, v.52, p.1453-1438, 1988.

QUIÑONES, O.R.G. et al. Características de solos construídos após mineração de carvão relacionadas ao processo de construção e a composição do material utilizado. Ciência Rural, Santa Maria, v.38, n.6, p.1564-1571, 2008. Disponível em <http:/ www.scielo.br/scielo.php? script $=$ sci arttext\&pid $=$ S0103$84782008000600012 \& \operatorname{lng}=$ pt\&nrm $=$ iso $>$. Acesso em: 29 mar. 2010. doi: $10.1590 / \mathrm{S} 0103-84782008000600012$.

SCHAFER, W.M. et al. Minesoil genesis and morphology in a spoil chronosequence in Montana. Soil Science Society of America Journal, Madison, v.44, p.802-807, 1980.

SHUKLA, M.K. et al. Physical and hydrological characteristics of reclaimed mine soils in Southeastern Ohio. Soil Science Society America Journal, Madison, v.68, p.1352-1359, 2004.

SIEGEL, S. Estatística não-paramétrica para as ciências do comportamento. São Paulo: McGraw-Hill do Brasil, 1975. $350 \mathrm{p}$.

SOARES, E.R. et al. Efeito da compactação e $\mathrm{CaCO}_{3}$ na oxidação da pirita em estéril de mineração de carvão. Revista Brasileira de Ciência do Solo, Viçosa, v.26, p.65-73, 2002.

STATISTIX 7. Analytical Software. User's manual. Tallahassee, 2000. 396p.

TEDESCO, J.M. et al. Análise de solo, plantas e outros materiais. 2.ed. Porto Alegre: Departamento de Solos, UFRGS, 1995. 174p. (Boletim técnico n.5). 